\title{
Effect of body weight on disability and chronic disease rates in the elderly in south-eastern Poland
}

\author{
Agnieszka Beata Ćwirlej-Sozańska ${ }^{1, A-F}{ }^{\oplus}$, Agnieszka Wiśniowska-Szurlej ${ }^{1, C-F \oplus,}$ \\ Anna Wilmowska-Pietruszyńska ${ }^{1, D-F}{ }^{\infty}$, Bernard Sozański ${ }^{1, C, E-F}{ }^{\oplus}$, Artur Mazur $^{1, C, E-F} \oplus$ \\ ${ }^{1}$ Department of Medicine, University of Rzeszow, Rzeszow, Poland \\ $A$ - Research concept and design, B - Collection and/or assembly of data, C - Data analysis and interpretation, \\ $D$ - Writing the article, E - Critical revision of the article, F - Final approval of article
}

\begin{abstract}
Ćwirlej-Sozańska AB, Wiśniowska-Szurlej A, Wilmowska-Pietruszyńska A, Sozański B, Mazur A. Effect of body weight on disability and chronic disease rates in the elderly in south-eastern Poland. Ann Agric Environ Med. 2020; 27(2): 240-247. doi: 10.26444/aaem/108542
\end{abstract}

\begin{abstract}
Introduction and objective. The rapid aging of the human population is an increasing challenge to public health. Effective strategies are required to prevent disability and dependency of the elderly. This study aimed to evaluate the effect of body weight on the prevalence of disability and chronic diseases among 60-80-year-old people living in south-eastern Poland. Materials and method. The study included 1,800 randomly selected people aged 60-80 years living in the Podkarpackie region of south-eastern Poland. Respondents, holders of a - personal identification number (PESEL), were randomly drawn by the Ministry of Interior and Administration (MSWiA) in Poland. The study was conducted in the form of a face-to-face interview at the respondent's residence. The WHODAS 2.0 questionnaire was used to assess disability and functioning. Socio-demographic data were also collected, and the body weight measured in $5 \%$ of the respondents after completion of the study. Statistical analysis was performed using Statistica 10.

Results. Respondents with Body Mass Index $(\mathrm{BMI})<18.5$ and $\mathrm{BMI} \geq 35.0$ had significantly higher disability levels than those in the normal weight and overweight categories. The greatest limitations were found in participating in everyday life, household activities, getting along and mobility. There was also a statistically significant relationship between BMI and the number of chronic diseases $(p<0.001)$.

Conclusions. When planning a healthcare strategy for people aged 60-80 living in Poland, additional support should be provided to those at risk in the categories of underweight and obesity. The obtained findings indicate that the health behaviour of seniors should be assessed - especially regarding their diet and eating habits, physical activity, and participation in social life - in order to tailor prevention programmes specifically to their needs.
\end{abstract}

\section{Key words}

aged, disability evaluation, chronic disease, body mass index

\section{INTRODUCTION}

Weight disorders, especially obesity and underweight, are a major public health problem in both developed and developing countries $[1,2]$. Excessive weight is a risk factor for the development of many chronic diseases [3], especially cardiovascular diseases [4], diabetes [5], musculoskeletal diseases [6], some respiratory diseases [7] and some types of cancer [8]. Underweight, in turn, is significantly associated in the case of the elderly with depression [9], dementia [10], frailty syndrome [11], cardiovascular and respiratory disorders [12], as well as with morbidity and complications after surgical interventions [13]. Previous research indicates that both obesity and underweight are associated with a lower quality of life [14] and greater mortality among older people $[15,16]$.

The World Health Organization (WHO) defines the healthy adult body mass range as Body Mass Index (BMI) (in $\mathrm{kg} / \mathrm{m}^{2}$ ) between $18.5-24.9$, based on reduced mortality risk [17]. However, the established range is predominantly based on studies regarding younger adults [18]. Research

Address for correspondence: Agnieszka Beata Ćwirlej-Sozańska, Department of Medicine, Institute of Physiotherapy, University of Rzeszow, Rzeszow, Poland, Hoffmanowej 25, 35-310 Rzeszów, Poland

E-mail: sozanska@ur.edu.pl

Received: 12.01.2019; accepted: 13.04.2019; first published: 30.04.2019 reviews considering BMI and mortality in older adults indicate that people with BMI determined as overweight, especially in the range of $25.0-27.0 \mathrm{~kg} / \mathrm{m}^{2}$ [19], or even $25.0-29.9 \mathrm{~kg} / \mathrm{m}^{2}[20]$ had a similar or lower risk of death from any cause than people with normal weight. Numerous studies have recorded a higher risk of morbidity in old age with an significant increase in BMI, and mortality with a decrease in this rate [21]. Regarding Polish research, there are only a few studies analyzing the relationship between individual BMI ranges among a representative population of older people [22]. To the best of the knowledge of the authors of this study, multidimensional analysis of the relationship between psychosocial functioning of older people, based on the International Classification of Functioning, Disability and Health (ICF) model and BMI, has been conducted todate. Therefore, the study aimed to evaluate the effect of body weight on the incidence of disability and chronic diseases among people aged 60-80 living in south-eastern Poland.

\section{MATERIALS AND METHOD}

Study subjects and design. The study was conducted in a randomly selected group of 1,800 people aged $60-80$ years living in the Podkarpackie region of south-eastern Poland. 


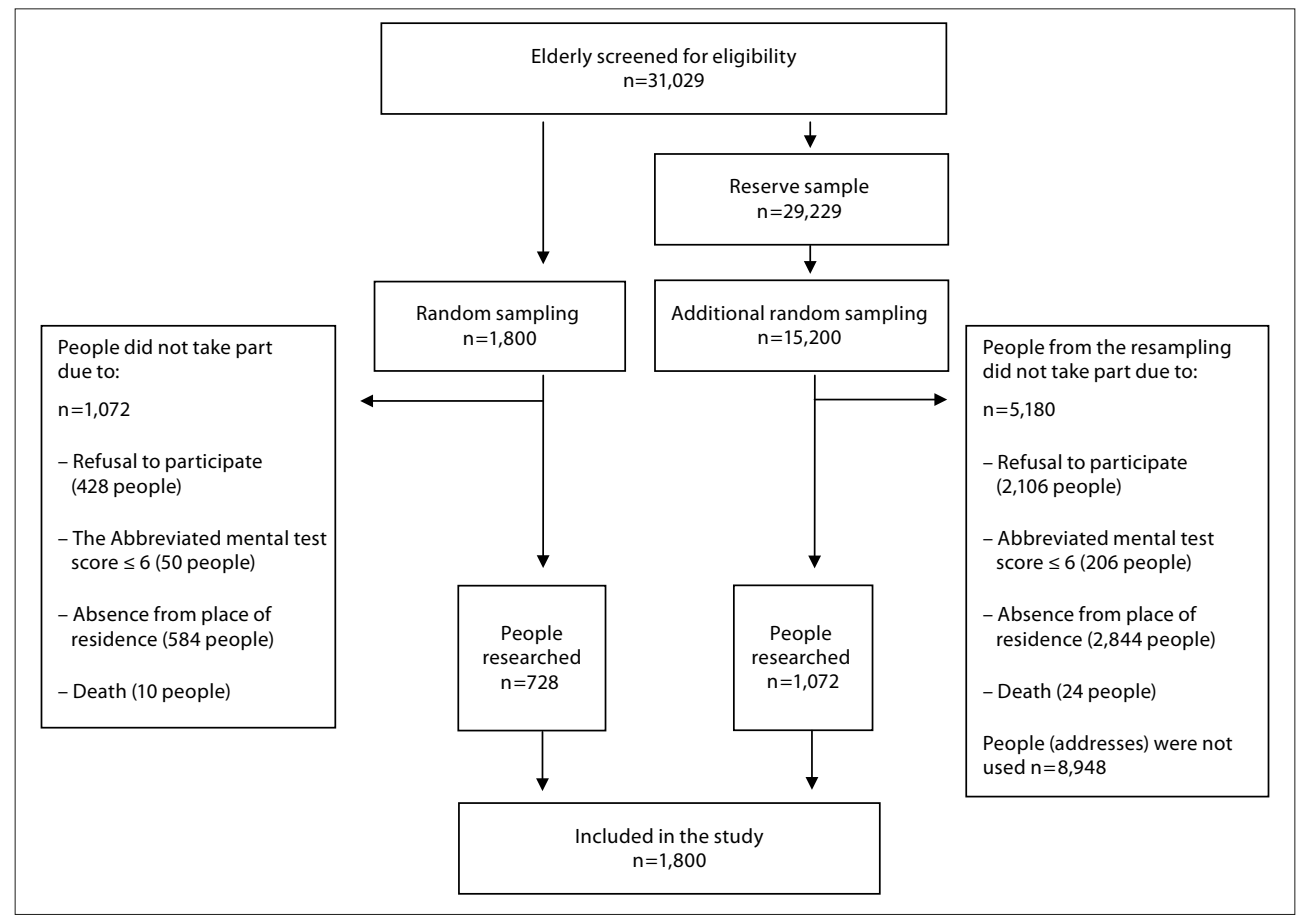

Figure 1. Flow diagram for respondents aged $60-80$ years in south-eastern Poland

From the database collected by the Ministry of the Interior and Administration (MSWiA) in Poland, a total of 31,029 people were drawn (name, address, and PESEL - personal identification number), including 6,029 people aged 60-70 and 25,000 aged 71-80. The reserve sample was 5,029 and 24,200 persons, respectively (Fig. 1).

Random sampling for each age group was made using SPSS programme version 23, without returning to the pool of already drawn respondents. This sample design ensured that the study was of high standard and representative of the population living in the Podkarpackie region of southeastern Poland. The assumed confidence interval (CI) was $95 \%$ (0.95) with an error estimate (maximum error) of $3 \%$.

The study was conducted by means of face-to-face interviews using the pen-and-paper method. To confirm the accuracy of the data on weight obtained in the interview, $5 \%$ of the subjects were subsequently weighed using the TANITA BC-731 scale. Criteria for inclusion in the study group were: age 60-80, cognitive status enabling a reliable interview (abbreviated mental test score [AMTS] $>6$ points used as a preliminary screening test), and informed consent to participate in the study.

The research was carried out by appropriately prepared and trained interviewers at the respondents' place of residence. According to the Declaration of Helsinki, study participants were informed about the purpose and course of the study, and gave their informed consent to participate. The results obtained, due to their representative nature, can be extrapolated to a wider population. The research project was approved by the Bioethics Committee of the University of Rzeszow.

Research tools. The research tools were the WHO Disability Assessment Schedule (WHODAS 2.0) and a questionnaire containing socio-demographic information which include: age, gender, place of residence, marital status, education, and income. The WHODAS 2.0 questionnaire is used to measure functioning, disability, and health, developed on the basis of the International Classification of Functioning, Disability and Health (ICF). It examines the level of functioning in six domains: Cognition (Domain 1), Mobility (Domain 2), Self-care (Domain 3), Getting along (Domain 4), Life activities: Household (Domain 5.1), and Participation in social life (Domain 6)[23]. Due to the low number of working people, Domain 5.2 (Life activities: work and school) was not analyzed in this study. The scale assigned to each question ranged from 1-5, where (1) indicated "no" difficulties, (2) "mild”, (3) "moderate", (4) “severe", and (5) "extreme" difficulties. According to the instruction manual, the multi-sectional positions were coded and the original score converted to a scale ranging from $0-100$, in which higher scores indicated more limitations $(0=$ no difficulty; $100=$ very high degree of difficulty). Finally, to determine the overall level of disability in the specific domains of WHODAS 2.0, the following ICF-compliant scale was used: no disability (0-4\%), mild disability (5-24\%), moderate disability (25-49\%), severe disability 50-95\%), and extreme disability (96-100\%) [24].

During the study, anthropometric data, height and weight were also collected, on which the BMI was calculated. To assess the accuracy of the collected data, weight measurement in 5\% of the study group was also performed after the interview. No statistically significant difference was found between the data collected in the interview, and the population weight measurement after interview ( $p=0.065)$, thus confirming the reliability of the collected data.

The BMI categories developed by the World Health Organization (WHO, Geneva, Switzerland) were used [25], and the following categories implemented: underweight $(\mathrm{BMI}<18.5)$, normal range $(18.5 \leq \mathrm{BMI}<25.0)$, grade I overweight $(25.0 \leq \mathrm{BMI}<27.5)$, grade II overweight $(27.5 \leq \mathrm{BMI}<30.0)$, obese class I $(30.0 \leq \mathrm{BMI}<35.0)$, and obese class II or more (BMI $\geq 35.0)$. 
Statistical analysis. The obtained data were analyzed using Statistica (version 10) software. Descriptive statistics were used for preliminary data analysis, Shapiro-Wilk test to test the normality of the distribution of variables, and the $\mathrm{t}$ test for dependent variables (due to the normal distribution of weight) was used to determine the concordance of the declared body weight with its actual value. Due to the lack of normal distribution of the studied variables, nonparametric tests, Mann-Whitney U test and Kruskal-Wallis test, were used to analyze the degree of disability in the individual BMI subgroups. When there were statistically significant differences in the compared subgroups, a multiple comparisons analysis was also performed. A statistical significance level of $\mathrm{p}<0.05$ was used.

On the basis of raw data, mean BMI values were determined in each of the following ranges: $\mathrm{BMI}<18.5,18.5 \leq \mathrm{BMI}<25.0$, $25.0 \leq \mathrm{BMI}<27.5,27.5 \leq \mathrm{BMI}<30.0,30.0 \leq \mathrm{BMI}<35.0, \mathrm{BMI} \geq 35.0$, and corresponding mean values of the disability level. For such observations carried out by the use of the classical method of least squares, the quadratic function describing the level of disability in relation to BMI was estimated. The quality of estimation was examined using the determination coefficient $\mathrm{R}^{2}$.

\section{RESULTS}

The study covered 1,800 people aged $60-80$ years, including 1,032 women and 768 men. The mean age of the tested group was 69.60 years $(\mathrm{SD}=6.07)$. A total of 827 persons were urban dwellers and 973 lived rurally. The vast majority of respondents were married (67.72\%), the majority of respondents had primary (34.72\%) and vocational education (26.28\%). Most of the respondents who answered the question about monthly income per person in the household stated a sum of PLN 1001-2000 (38.72\%). The vast majority of the respondents presented body mass index in the range of $18.5 \leq \mathrm{BMI}<25.0(27.11 \%)$, followed by $25.0 \leq \mathrm{BMI}<27.5$ $(20.00 \%)$ and $27.5 \leq \mathrm{BMI}<30.0(23.61 \%)$; a large group were also in the range of $30.0 \leq \mathrm{BMI}<35.0$, $(19.78 \%)$. The least numerous group was represented by subjects with $\mathrm{BMI} \geq 35.0$ (6.94\%) and $\mathrm{BMI}<18.5$ (2.56\%) (Tab. 1).

A statistically significant relationship was observed between BMI and the level of disability ( $\mathrm{p}<0.001)$ (Tab. 2). Respondents with $\mathrm{BMI}<18.5$ had significantly higher disability levels than those with a range of $18.5 \leq \mathrm{BMI}<25.0(\mathrm{p}=0.004)$, $25.0 \leq \mathrm{BMI}<27.0(\mathrm{p}=0.001), 27.0 \leq \mathrm{BMI}<30.0(\mathrm{p}=0.001)$, and those with class I obesity $(\mathrm{p}=0.019)$. Similarly, it was found that respondents with of $\mathrm{BMI} \geq 35$ were characterized by a significantly higher level of disability than those with a range of $18.5 \leq \mathrm{BMI}<25.0(\mathrm{p}=0.006), 25.0 \leq \mathrm{BMI}<27.0(\mathrm{p}=0.001)$, $27.0 \leq \mathrm{BMI}<30.0(\mathrm{p}=0.001)$, and those with class I obesity $(\mathrm{p}=0.066)(\mathrm{Tab} .3)$.

Estimation of the mean level of disability in relation to the mean BMI values in individual categories was made on the basis of the classic least squares method. The function used was as follows: disability level $(0-100)=0.1099 \times \mathrm{BMI}^{2}-6.3487$ $\times \mathrm{BMI}+112.1\left(\mathrm{R}^{2}=0.9612\right)$. This indicated that the lowest overall level of disability in the study group of older people was at a BMI value of $28.88 \mathrm{~kg} / \mathrm{m}^{2}$ (Fig. 2).

In individual WHODAS 2.0 domains, the highest level of disability occurred among respondents with $\mathrm{BMI}<18.5$ and $\mathrm{BMI} \geq 35$. In each domain, statistically significant
Table 1. Socio-demographic characteristics of the study population

\begin{tabular}{|c|c|c|}
\hline Demographic characteristics $(\mathrm{N}=1,800)$ & $\begin{array}{l}\text { Number } \\
\text { (N) }\end{array}$ & $\begin{array}{c}\text { Percentage } \\
(\%)\end{array}$ \\
\hline \multicolumn{3}{|l|}{ 1. Gender } \\
\hline Female & 1,032 & 57.33 \\
\hline Male & 768 & 42.67 \\
\hline \multicolumn{3}{|l|}{ 2. Place of residence } \\
\hline Urban & 827 & 45.94 \\
\hline Rural & 973 & 54.06 \\
\hline \multicolumn{3}{|l|}{ 3. Marital status } \\
\hline Single & 74 & 4.11 \\
\hline Married & 1,219 & 67.72 \\
\hline Separated/divorced & 40 & 2.23 \\
\hline Widower/widow & 456 & 25.33 \\
\hline Living with a partner & 11 & 0.61 \\
\hline \multicolumn{3}{|l|}{ 4. Education } \\
\hline Primary & 625 & 34.72 \\
\hline Vocational & 473 & 26.28 \\
\hline Secondary comprehensive & 152 & 8.44 \\
\hline Secondary vocational & 355 & 19.73 \\
\hline Tertiary & 195 & 10.83 \\
\hline \multicolumn{3}{|l|}{ 5. Income per capita/month } \\
\hline Up to 1,000 PLN* & 177 & 9.83 \\
\hline 1,001 PLN-2,000 PLN & 697 & 38.72 \\
\hline 2,001 PLN-3,000 PLN & 253 & 14.06 \\
\hline 3,001 PLN-4,000 PLN & 91 & 5.06 \\
\hline 4,001 PLN and above & 60 & 3.33 \\
\hline No data & 522 & 29.00 \\
\hline \multicolumn{3}{|l|}{ 6. Body Mass Index } \\
\hline $\mathrm{BMI}<18.5$ & 46 & 2.56 \\
\hline $18.5 \leq \mathrm{BMI}<25.0$ & 488 & 27.11 \\
\hline $25.0 \leq \mathrm{BMI}<27.0$ & 360 & 20.00 \\
\hline $27.0 \leq \mathrm{BMI}<30.0$ & 425 & 23.61 \\
\hline $30.0 \leq \mathrm{BMI}<35.0$ & 356 & 19.78 \\
\hline $\mathrm{BMI} \geq 35.0$ & 125 & 6.94 \\
\hline \multicolumn{3}{|l|}{ 7. Disability (WHODAS 2.0) } \\
\hline None & 376 & 20.88 \\
\hline Mild & 723 & 40.17 \\
\hline Moderate & 496 & 27.56 \\
\hline Severe & 200 & 11.11 \\
\hline Extreme & 5 & 0.28 \\
\hline \multicolumn{3}{|l|}{ 8. No. of chronic diseases } \\
\hline 0 & 178 & 9.89 \\
\hline $1-2$ & 353 & 19.61 \\
\hline $3-4$ & 418 & 23.22 \\
\hline 5 or more & 851 & 47.28 \\
\hline
\end{tabular}

"PLN (Polish zloty) — official name of Polish currency

differences in disability levels were observed with respect to BMI ranges (Tab. 4).

In Domain 1, assessing cognitive function, statistically significant differences in disability levels were observed between respondents with $\mathrm{BMI}<18.5$ and those with ranges $18.5 \leq \mathrm{BMI}<25.0 \quad(\mathrm{p}=0.004), \quad 25.0 \leq \mathrm{BMI}<27.0$ 
Table 2. Disability level WHODAS 2.0 according to BMI ranges

\begin{tabular}{|c|c|c|c|c|c|c|}
\hline \multirow[b]{2}{*}{$\begin{array}{l}\text { Body Mass } \\
\text { Index ranges }\end{array}$} & \multicolumn{6}{|c|}{ Disability } \\
\hline & $\begin{array}{c}\text { Overall mean } \\
\pm \mathrm{SD} \\
(95 \% \mathrm{Cl})\end{array}$ & $\begin{array}{c}\text { None } \\
(\%)\end{array}$ & $\begin{array}{l}\text { Mild } \\
(\%)\end{array}$ & $\begin{array}{l}\text { Moderate } \\
(\%)\end{array}$ & $\begin{array}{l}\text { Severe } \\
(\%)\end{array}$ & $\begin{array}{c}\text { Extreme } \\
\text { high } \\
(\%)\end{array}$ \\
\hline $\mathrm{BMI}<18.5$ & $\begin{array}{l}33.60 \pm 22.03 \\
(27.06-40.14)\end{array}$ & 4.75 & 34.78 & 36.96 & 23.91 & 0.00 \\
\hline $18.5 \leq \mathrm{BMI}<25$ & $\begin{array}{l}22.53 \pm 21.81 \\
(20.59-24.47)\end{array}$ & 22.13 & 39.96 & 26.43 & 11.07 & 0.41 \\
\hline $25 \leq \mathrm{BMI}<27$ & $\begin{array}{l}20.84 \pm 20.27 \\
(18.74-22.94)\end{array}$ & 29.72 & 34.17 & 26.39 & 9.72 & 0.00 \\
\hline $27 \leq \mathrm{BMI}<30$ & $\begin{array}{l}20.82 \pm 19.53 \\
(18.96-22.68)\end{array}$ & 25.65 & 38.12 & 26.82 & 9.18 & 0.24 \\
\hline $30 \leq \mathrm{BMI}<35$ & $\begin{array}{l}22.93 \pm 20.30 \\
(20.81-25.05)\end{array}$ & 17.70 & 42.13 & 28.37 & 11.80 & 0.00 \\
\hline $\mathrm{BMI} \geq 35$ & $\begin{array}{l}29.70 \pm 23.52 \\
(25.53-33.86)\end{array}$ & 8.80 & 42.40 & 32.00 & 15.20 & 1.60 \\
\hline$p$ & \multicolumn{6}{|c|}{$<0.001$} \\
\hline
\end{tabular}

$\mathrm{Cl}$ - confidence interval; SD - standard deviation

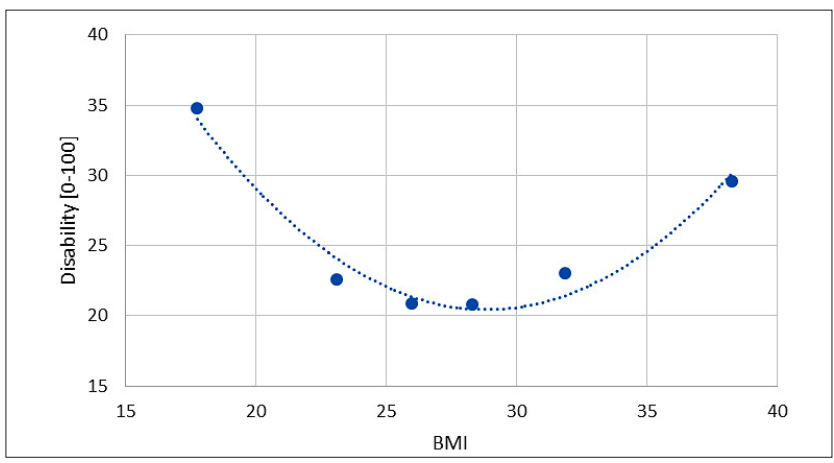

Figure 2. Relationship between mean BMI values and general disability level

$(\mathrm{p}=0.002), 27.0 \leq \mathrm{BMI}<30.0(\mathrm{p}=0.003)$, and respondents with $30.0 \leq \mathrm{BMI}<35.0(\mathrm{p}=0.018)$, as well as between respondents with $\mathrm{BMI} \geq 35.0$ and those with ranges $18.5 \leq \mathrm{BMI}<25.0$ $(\mathrm{p}=0.041), 25.0 \leq \mathrm{BMI}<27.0(\mathrm{p}=0.012), 27.0 \leq \mathrm{BMI}<30.0$ $(\mathrm{p}=0.028)$ (Tab. 3).

In Domain 2, assessing movement difficulties, statistically significant differences were observed between respondents with $\mathrm{BMI} \geq 35$ and those with ranges $18.5 \leq \mathrm{BMI}<25.0$ $(\mathrm{p}=0.001), 25.0 \leq \mathrm{BMI}<27.0(\mathrm{p}<0.001), 27.0 \leq \mathrm{BMI}<30.0$ $(\mathrm{p}<0.001), 30.0 \leq \mathrm{BMI}<35.0(\mathrm{p}=0.020)$ (Tab. 3).

In Domain 3, assessing difficulties in self-care, statistically significant differences were observed in respondents with $\mathrm{BMI}<18.5$ and those with ranges $18.5 \leq \mathrm{BMI}<25.0(\mathrm{p}=0.012)$, $25.0 \leq \mathrm{BMI}<27.0(\mathrm{p}=0.009), 27.0 \leq \mathrm{BMI}<30.0(\mathrm{p}=0.003)$, and $30.0 \leq \mathrm{BMI}<35.0(\mathrm{p}=0.005)$. Statistically significant differences were also found between respondents with BMI $\geq 35$ and those with ranges $18.5 \leq \mathrm{BMI}<25.0(\mathrm{p}=0.019), 25.0 \leq \mathrm{BMI}<27.0$ $(\mathrm{p}=0.015), 27.0 \leq \mathrm{BMI}<30.0(\mathrm{p}=0.002)$, and $30.0 \leq \mathrm{BMI}<35.0$ $(\mathrm{p}=0.007)($ Tab. 3).

In Domain 4, assessing getting along with other people, statistically significant differences were observed between respondents with $\mathrm{BMI}<18.5$ and those with range $27.0 \leq \mathrm{BMI}<30.0(\mathrm{p}=0.029)$ (Tab. 3).

In Domain 5.1, assessing difficulties in daily household activities, statistically significant differences were observed between respondents with $\mathrm{BMI}<18.5$ and those with ranges $18.5 \leq \mathrm{BMI}<25.0 \quad(\mathrm{p}=0.003), 25.0 \leq \mathrm{BMI}<27.0 \quad(\mathrm{p}=0.001)$, $27.0 \leq \mathrm{BMI}<30.0(\mathrm{p}=0.001)$, and $30.0 \leq \mathrm{BMI}<35.0(\mathrm{p}=0.013)$.
Table 3. BMI ranges and level of disability according to WHODAS 2.0 ( $p$ value for multiple comparisons - post hoc analysis)

\begin{tabular}{|c|c|c|c|c|c|c|}
\hline WHODAS 2.0 & $\begin{array}{c}\text { BMI } \\
<18.5 \\
\end{array}$ & $\begin{array}{c}18.5 \leq \\
\mathrm{BMI}<25\end{array}$ & $\begin{array}{c}25 \leq \\
\mathrm{BMI}<27\end{array}$ & $\begin{array}{c}27 \leq \\
\mathrm{BMI}<30\end{array}$ & $\begin{array}{c}30 \leq \\
\mathrm{BMI}<35\end{array}$ & $\begin{array}{l}\text { BMI } \\
\geq 35\end{array}$ \\
\hline \multicolumn{7}{|l|}{ Total WHODAS } \\
\hline $\mathrm{BMI}<18.5$ & & 0.004 & 0.001 & 0.001 & 0.019 & 1.000 \\
\hline $18.5 \leq \mathrm{BMI}<25$ & 0.004 & & 1.000 & 1.000 & 1.000 & 0.006 \\
\hline $25 \leq \mathrm{BMI}<27$ & 0.001 & 1.000 & & 1.000 & 1.000 & 0.001 \\
\hline $27 \leq \mathrm{BMI}<30$ & 0.001 & 1.000 & 1.000 & & 1.000 & 0.001 \\
\hline $30 \leq \mathrm{BMI}<35$ & 0.019 & 1.000 & 1.000 & 1.000 & & 0.066 \\
\hline$\underline{B M I} \geq 35$ & 1.000 & 0.006 & 0.001 & 0.001 & 0.066 & \\
\hline \multicolumn{7}{|l|}{$\begin{array}{l}\text { WHODAS } \\
\text { domains }\end{array}$} \\
\hline \multicolumn{7}{|l|}{$\begin{array}{l}\text { Domain } 1 \\
\text { Cognition }\end{array}$} \\
\hline BMI $<18.5$ & & 0.004 & 0.002 & 0.003 & 0.018 & 1.000 \\
\hline $18.5 \leq \mathrm{BMI}<25$ & 0.004 & & 1.000 & 1.000 & 1.000 & 0.041 \\
\hline $25 \leq \mathrm{BMI}<27$ & 0.002 & 1.000 & & 1.000 & 1.000 & 0.012 \\
\hline $27 \leq \mathrm{BMI}<30$ & 0.003 & 1.000 & 1.000 & & 1.000 & 0.028 \\
\hline $30 \leq \mathrm{BMI}<35$ & 0.018 & 1.000 & 1.000 & 1.000 & & 0.250 \\
\hline $\mathrm{BMI} \geq 35$ & 1.000 & 0.041 & 0.012 & 0.028 & 0.250 & \\
\hline
\end{tabular}

Domain 2

Mobility

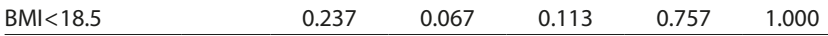

\begin{tabular}{llllll}
\hline $18.5 \leq \mathrm{BMI}<25$ & 0.237 & 1.000 & 1.000 & 1.000 & 0.001 \\
\hline
\end{tabular}

\begin{tabular}{llllll}
\hline $25 \leq \mathrm{BMI}<27$ & 0.067 & 1.000 & 1.000 & 0.955 & $<0.001$ \\
\hline $27 \leq \mathrm{BMI}$ &
\end{tabular}

\begin{tabular}{llllll}
\hline $27 \leq \mathrm{BMI}<30$ & 0.113 & 1.000 & 1.000 & 1.000 & $<0.001$ \\
\hline $30 \leq \mathrm{BMI}$
\end{tabular}

\begin{tabular}{llllll}
\hline $30 \leq \mathrm{BMI}<35$ & 0.757 & 1.000 & 0.955 & 1.000 & 0.020 \\
\hline $\mathrm{BMI}$
\end{tabular}

$\begin{array}{llllll}\mathrm{BMI} \geq 35 & 1.000 & 0.001 & <0.001 & <0.001 & 0.020\end{array}$

Domain 3

Self-care

$\begin{array}{llllll}\mathrm{BMI}<18.5 & 0.012 & 0.009 & 0.003 & 0.005 & 1.000\end{array}$

\begin{tabular}{llllll}
\hline $18.5 \leq \mathrm{BMI}<25$ & 0.012 & 1.000 & 1.000 & 1.000 & 0.019 \\
\hline
\end{tabular}

\begin{tabular}{llllll}
$25 \leq \mathrm{BMI}<27$ & 0.009 & 1.000 & 1.000 & 1.000 & 0.015 \\
\hline
\end{tabular}

\begin{tabular}{llllll}
\hline $27 \leq \mathrm{BMI}<30$ & 0.003 & 1.000 & 1.000 & 1.000 & 0.002 \\
\hline
\end{tabular}

\begin{tabular}{llllll}
\hline $30 \leq \mathrm{BMI}<35$ & 0.005 & 1.000 & 1.000 & 1.000 & 0.007 \\
\hline
\end{tabular}

\begin{tabular}{llllll}
$\mathrm{BMI} \geq 35$ & 1.000 & 0.019 & 0.015 & 0.002 & 0.007 \\
\hline
\end{tabular}

Domain 4

Getting along

\begin{tabular}{lllllll}
$\mathrm{BMI}<18.5$ & & 0.060 & 0.060 & 0.029 & 0.134 & 1.000 \\
\hline $18.5 \leq \mathrm{BMI}<25$ & 0.060 & & 1.000 & 1.000 & 1.000 & 1.000 \\
\hline $25 \leq \mathrm{BMI}<27$ & 0.060 & 1.000 & & 1.000 & 1.000 & 1.000 \\
\hline $27 \leq \mathrm{BMI}<30$ & 0.029 & 1.000 & 1.000 & & 1.000 & 0.584 \\
\hline $30 \leq \mathrm{BMI}<35$ & 0.134 & 1.000 & 1.000 & 1.000 & & 1.000 \\
\hline $\mathrm{BMI} \geq 35$ & 1.000 & 1.000 & 1.000 & 0.584 & 1.000 & \\
\hline
\end{tabular}

Domain 5.1

Life activities: household

\begin{tabular}{lllllll}
\hline $\mathrm{BMl}<18.5$ & & 0.003 & 0.001 & 0.001 & 0.013 & 1.000 \\
\hline $18.5 \leq \mathrm{BMI}<25$ & 0.003 & & 1.000 & 1.000 & 1.000 & 0.086 \\
\hline $25 \leq \mathrm{BMI}<27$ & 0.001 & 1.000 & & 1.000 & 1.000 & 0.023 \\
\hline $27 \leq \mathrm{BMI}<30$ & 0.001 & 1.000 & 1.000 & & 1.000 & 0.020 \\
\hline $30 \leq \mathrm{BMI}<35$ & 0.013 & 1.000 & 1.000 & 1.000 & & 0.447 \\
\hline $\mathrm{BMI} \geq 35$ & 1.000 & 0.086 & 0.023 & 0.020 & 0.447 & \\
\hline
\end{tabular}

Domain 6

Participation

\begin{tabular}{lcccccc}
\hline $\mathrm{BMI}<18.5$ & & 0.237 & 0.054 & 0.082 & 0.788 & 1.000 \\
\hline $18.5 \leq \mathrm{BMI}<25$ & 0.237 & & 1.000 & 1.000 & 1.000 & 0.006 \\
\hline $25 \leq \mathrm{BMI}<27$ & 0.054 & 1.000 & & 1.000 & 0.619 & $<0.001$ \\
\hline $27 \leq \mathrm{BMI}<30$ & 0.082 & 1.000 & 1.000 & & 1.000 & 0.001 \\
\hline $30 \leq \mathrm{BMI}<35$ & 0.788 & 1.000 & 0.619 & 1.000 & & 0.086 \\
\hline $\mathrm{BMI} \geq 35$ & 1.000 & 0.006 & $<0.001$ & 0.001 & 0.086 & \\
\hline
\end{tabular}


Table 4. Disability level WHODAS 2.0 domains according to BMI ranges

\begin{tabular}{|c|c|c|c|c|c|c|}
\hline \multirow[b]{2}{*}{$\begin{array}{l}\text { Body Mass Index } \\
\text { ranges }\end{array}$} & \multicolumn{6}{|c|}{ Disability domains } \\
\hline & $\begin{array}{l}\text { Domain } 1 \\
\text { Cognition }\end{array}$ & $\begin{array}{l}\text { Domain } 2 \\
\text { Mobility }\end{array}$ & $\begin{array}{l}\text { Domain } 3 \\
\text { Self-care }\end{array}$ & $\begin{array}{l}\text { Domain } 4 \\
\text { Getting along }\end{array}$ & $\begin{array}{c}\text { Domain } 5.1 \\
\text { Life activities: household }\end{array}$ & $\begin{array}{c}\text { Domain } 6 \\
\text { Participation }\end{array}$ \\
\hline $\mathrm{BMI}<18.5$ & $\begin{array}{l}27.93 \pm 21.36 \\
(21.59-34.28)\end{array}$ & $\begin{array}{l}34.92 \pm 29.00 \\
(26.31-43.53)\end{array}$ & $\begin{array}{l}22.83 \pm 26.47 \\
(14.97-30.69)\end{array}$ & $\begin{array}{l}35.14 \pm 30.83 \\
(25.99-44.30)\end{array}$ & $\begin{array}{l}44.78 \pm 29.27 \\
(36.09-53.47)\end{array}$ & $\begin{array}{l}36.50 \pm 24.93 \\
(29.10-43.91)\end{array}$ \\
\hline $18.5 \leq \mathrm{BMI}<25$ & $\begin{array}{l}17.95 \pm 22.61 \\
(15.94-19.96)\end{array}$ & $\begin{array}{l}24.78 \pm 28.01 \\
(22.29-27.27)\end{array}$ & $\begin{array}{l}11.02 \pm 21.05 \\
(9.15-12.90)\end{array}$ & $\begin{array}{l}22.37 \pm 26.12 \\
(20.05-24.69)\end{array}$ & $\begin{array}{l}27.85 \pm 30.15 \\
(25.17-30.53)\end{array}$ & $\begin{array}{l}27.48 \pm 22.83 \\
(25.45-29.52)\end{array}$ \\
\hline $25 \leq \mathrm{BMl}<27$ & $\begin{array}{l}16.71 \pm 21.22 \\
(14.51-18.91)\end{array}$ & $\begin{array}{l}22.59 \pm 26.15 \\
(19.88-25.30)\end{array}$ & $\begin{array}{l}9.08 \pm 16.92 \\
(7.33-10.84)\end{array}$ & $\begin{array}{l}21.30 \pm 23.92 \\
(18.82-23.78)\end{array}$ & $\begin{array}{l}25.89 \pm 28.53 \\
(22.93-28.85)\end{array}$ & $\begin{array}{l}25.69 \pm 22.45 \\
(23.37-28.02)\end{array}$ \\
\hline $27 \leq \mathrm{BMI}<30$ & $\begin{array}{l}16.84 \pm 20.97 \\
(14.84-18.83)\end{array}$ & $\begin{array}{l}22.99 \pm 25.53 \\
(20.55-25.42)\end{array}$ & $\begin{array}{l}8.66 \pm 17.23 \\
(7.02-10.30)\end{array}$ & $\begin{array}{l}20.49 \pm 23.74 \\
(18.23-22.75)\end{array}$ & $\begin{array}{l}25.74 \pm 28.91 \\
(22.98-28.50)\end{array}$ & $\begin{array}{l}25.87 \pm 21.72 \\
(23.80-27.94)\end{array}$ \\
\hline $30 \leq \mathrm{BMI}<35$ & $\begin{array}{l}17.70 \pm 20.73 \\
(15.54-19.86)\end{array}$ & $\begin{array}{l}26.00 \pm 27.06 \\
(23.18-28.82)\end{array}$ & $\begin{array}{l}8.79 \pm 17.23 \\
(7.00-10.59)\end{array}$ & $\begin{array}{l}22.82 \pm 25.57 \\
(20.16-25.49)\end{array}$ & $\begin{array}{l}28.62 \pm 29.09 \\
(25.59-31.66)\end{array}$ & $\begin{array}{l}28.82 \pm 22.74 \\
(26.44-31.19)\end{array}$ \\
\hline $\mathrm{p}$ & $<0.001$ & $<0.001$ & $<0.001$ & 0.017 & $<0.001$ & $<0.001$ \\
\hline
\end{tabular}

$\mathrm{Cl}$ - confidence interval; SD - standard deviation

Statistically significant differences were also found between respondents with $\mathrm{BMI} \geq 35.0$ and ranges $25.0 \leq \mathrm{BMI}<27.0$ $(\mathrm{p}=0.023), 27.0 \leq \mathrm{BMI}<30.0(\mathrm{p}=0.020)($ Tab. 3).

In Domain 6, assessing participation in social life, statistically significant differences were observed between respondents with $\mathrm{BMI} \geq 35.0$ and those with $18.5 \leq \mathrm{BMI}<25.0$ $(\mathrm{p}=0.006), 25.0 \leq \mathrm{BMI}<27.0(\mathrm{p}<0.001)$ and $27.0 \leq \mathrm{BMI}<30.0$ $(\mathrm{p}=0.001)$. Statistically significant differences were also found between respondents with ranges $18.5 \leq \mathrm{BMI}<25.0(\mathrm{p}=0.006)$, $25.0 \leq \mathrm{BMI}<27.0(\mathrm{p}<0.001), 27.0 \leq \mathrm{BMI}<30.0(\mathrm{p}=0.001)$ and $\mathrm{BMI} \geq 35.0$ (Tab. 3 ).

A statistically significant relationship between BMI and the number of chronic diseases $(\mathrm{p}<0.001)$ was also found. More than half of people with $\mathrm{BMI}<18.5$ and $\mathrm{BMI} \geq 35.0$, had five or more chronic diseases (Tab. 5).

\section{DISCUSSION}

The results obtained in the current study provide reliable information on the relationship between BMI and disability and chronic diseases among 60-80-year-olds living in southeastern Poland. By investigating a randomly selected group of 1,800 older people, it was confirmed that the vast majority of respondents were overweight, including $20.00 \%$ in the range $25.0 \leq \mathrm{BMI}<27.0$ and $23.61 \%$ in the range $27.0 \leq \mathrm{BMI}<30.0$. The second largest group were respondents with normal body weight $(27.11 \%)$, whereas the next largest group were those with class I obesity (19.78\%). On the other hand, older people in class II obesity constituted almost 7\% of the studied population; while considering the underweight category they amounted over $2.5 \%$. Similar results were obtained in the PolSenior study performed on a representative group of the elderly in Poland, where the underweight category was found in $1.5 \%$ of respondents $(1.4 \%$ of women and $1.6 \%$ of men), normal body weight in $25.8 \%$ (23.2\% of women and $28.2 \%$ of men), overweight in $40.8 \%$ (36.4\% of women and $44.6 \%$ of men), obesity in $31.9 \%$ (39.0\% of women and $25.6 \%$ of men), including severe obesity in $2.0 \%$ (3.4\% women and $0.8 \%$ men) [22]

It was found that the incidence of class II obesity and greater $(\mathrm{BMI} \geq 35.0)$ or underweight $(\mathrm{BMI}<18.5)$ was significantly associated with higher levels of disability, compared to those of the same age with normal body weight or overweight range. The greatest limitations were found in the elderly with extremely high or low body weight in each of the studied domains of functioning. The highest number of chronic diseases was also observed in people with $\mathrm{BMI}<18.5$ and $\mathrm{BMI} \geq 35.0$.

It seems that few studies have analyzed the relationship between body weight of the elderly and disability using a multidimensional approach [26]. To date, only a few publications have assessed the impact of obesity on the prevalence of disability [27], most of which have focused on disability in performing basic and complex daily activities [28], or on the mobility of the elderly [29]. Corona et al. stated that both too high and too low body weight are associated with an increased risk of disability development

Table 5. Number of chronic diseases according to BMI ranges

\begin{tabular}{|c|c|c|c|c|c|c|}
\hline \multirow[b]{2}{*}{ No. of diseases } & \multicolumn{6}{|c|}{ Body Mass Index ranges } \\
\hline & $\begin{array}{c}\mathrm{BMI}<18.5 \\
\mathrm{~N}(\%)\end{array}$ & $\begin{array}{c}18.5 \leq \mathrm{BMI}<25 \\
\mathrm{~N}(\%)\end{array}$ & $\begin{array}{c}25 \leq \mathrm{BMI}<27 \\
\mathrm{~N}(\%)\end{array}$ & $\begin{array}{c}27 \leq \mathrm{BMI}<30 \\
\mathrm{~N}(\%)\end{array}$ & $\begin{array}{c}30 \leq \mathrm{BMI}<35 \\
\mathrm{~N}(\%)\end{array}$ & $\begin{array}{c}\mathrm{BMI} \geq 35 \\
\mathrm{~N}(\%)\end{array}$ \\
\hline $1-2$ & $8(17.39)$ & $92(18.85)$ & $70(19.44)$ & $81(19.06)$ & $83(23.31)$ & $19(15.20)$ \\
\hline $3-4$ & $12(26.09)$ & $117(23.98)$ & $82(22.78)$ & $106(24.94)$ & $70(19.66)$ & $31(24.80)$ \\
\hline
\end{tabular}


in relation to the Instrumental Activities of Daily Living Scale (IADL) among the elderly, regardless of other risk factors [30]. They also identified significant correlations between functional limitations in older people with obesity and underweight categories [31]. It is worth mentioning that quite a lot of research mentioned the negative effect of obesity on the physical capability of older people. De Stefano et al. conducted a study among 3,000 older people and demonstrated that obese persons were characterized by poor physical performance [32]. Germain et al. pointed out that obesity and muscle weakness are associated with functional disability in older people [33]. Obesity is associated with a lower perception of one's own health and an increased risk of disability [34]. The findings of the current study confirm data from a 12-year Canadian National Population Health Survey in which it was found that patients with obesity had double the risk of disability [35]. The current results are also consistent with those of Buttery et al., who analyzed changes in the physical functioning of older people from 1997-1999 and from 2008-2011, and found that the decline in physical functioning was greater in people with obesity, compared to those with normal body weight [36]. Likewise, Backholer et al. showed an increased risk of disability in people aged over 65 years with grade I and II obesity, compared to those with normal body weight [37]. Furthermore, according to Wong et al., reducing the incidence of obesity can reduce the incidence of disability in older people by nearly $26 \%$ [38].

The presented study additionally shows that very low body mass is also associated with a significantly higher incidence of disability in the elderly. Maeda et al. indicated a significant relationship between the underweight category and mobility limitations [39]. Zunzunegui et al. stressed that being underweight is a significant risk factor for mortality among older people, whereas overweight and mild obesity are associated with the lowest mortality, especially among men and people with cardiovascular disease [40]. Rejewski et al. showed that underweight and obesity increase the risk of disability, whereas overweight may have protective effects [41].

In the current study, the lowest level of disability was identified for a BMI of $28.88 \mathrm{~kg} / \mathrm{m}^{2}$. Grzegorzewska et al. also presented that the occurrence of overweight and mild obesity in the elderly population was associated with better functional performance [21], whereas Lv et al. pointed out that the occurrence of overweight among older people significantly reduced the risk of disability in everyday life. The authors suggested that current BMI data might require re-evaluation in order to estimate accurately the risk of functional disability and mortality in the elderly population [42].

In the presented multidimensional assessment of disability with WHODAS 2.0, it was found that the elderly with $\mathrm{BMI}<18.5$ and $\mathrm{BMI} \geq 35.0$ were characterized by higher average levels of disability in all domains studied in relation to people in the range of $25.0 \leq \mathrm{BMI}<30.0$. The highest disability rate was found in mobility, household activities, getting along, and participation in social life. Both underweight category and obesity were associated with reduced activity and daily activities, as discussed above. A further consequence of body weight disorders was limited participation in everyday life. Older people with obesity or underweight were significantly more likely to stay at home and were lonely [43]. Obesity is associated with a feeling of exclusion from socio-cultural life [44]. Oliviera et al. showed that people with obesity more often experienced a lack of social support and loneliness [45].

The results of the presented study confirm the relationship between BMI and a number of chronic conditions, which identified that people with $\mathrm{BMI}<18.5$ and $\mathrm{BMI} \geq 35.0$ were significantly more likely to suffer from five or more chronic diseases, which is consistent with previous reports [46]; for example, Kearns et al. presented that the incidence of chronic diseases increases with growing BMI [47]. The authors also pointed out that the reduction in BMI by one unit $\left(1 \mathrm{~kg} / \mathrm{m}^{2}\right)$ across the entire population for both genders may reduce the number of chronic diseases by 26-28 cases per 1,000 men and women. Similarly, the results of the analysis of 97 prospective cohorts with 1.8 million participants, indicate that in patients with increased body weight, the risk of coronary heart disease was increased by $46 \%$ and the risk of stroke by $76 \%$ [48]. According to Dhana et al., the increase in incidence of obesity and progress in cardiovascular disease (CVD) treatment will increase the proportion of people living with CVD over a longer period of time. This will be reflected in the increase in health care costs and deterioration in the quality of life of older people [49]. On the other hand, the underweight category is significantly associated with the incidence of cognitive function disorders in the elderly [10] and the frailty syndrome [11].

In the current study, it was also found that slightly elevated BMI (within the limits of overweight, $25.0 \leq \mathrm{BMI}<30.0$ ) in the elderly is associated with lower levels of disability and lower number of chronic diseases, to a similar level to normal BMI. Flicker et al. reported similar findings revealing that the overweight elderly were less likely to die or to have many chronic conditions, while extremely low weight and obesity in the elderly are more dangerous [50].

Advantages and limitations of the study. This is the first population study in Eastern Europe to analyze accurately the association of BMI with disability using a multidimensional approach in people aged $60-80$ years. The obtained results provide valuable information on the relationship between $\mathrm{BMI}$ and the occurrence of disability in various domains of human functioning. The limitation of the work is that the study was conducted only in the Podkarpackie region of south-eastern Poland. However, to some degree, the results can be extrapolated to the entire population of Poland. The study should be extended both in terms of the sample (i.e., to the whole territory of Poland) and the research tools (i.e., extend the anthropometric measurements, analysis of eating habits, and physical activity of the elderly).

Practical implications. The obtained results indicate that health policy programmes in Poland should incorporate support for elderly people at risk of underweight and obesity ranges, and include a detailed assessment of the health behaviour of seniors. The results also revealed that older people with $\mathrm{BMI}$ in the range of $18.5 \leq \mathrm{BMI}<30.0$ were characterized by an overall lower level of disability than in the other BMI ranges, and the lowest level of disability was recorded for BMI $28.88 \mathrm{~kg} / \mathrm{m}^{2}$. The study proves also that underweight and obesity are the main factors contributing to limiting independence, activity, and participation in family and social life in the elderly. Obesity and underweight are also closely related to a significantly higher incidence of chronic diseases; therefore, achieving a relatively small reduction in BMI in the obese elderly population, or increasing BMI 
ranges in underweight persons, may reduce the level of psychophysical disability and alleviate the burden of chronic diseases. It is therefore necessary to extend research into the relationship between disability and extreme body weight to examine also nutrition and levels of physical activity. This will allow researchers to prepare appropriate prevention programmes tailored to the needs of the elderly.

\section{Consent for publication}

Not applicable.

\section{Competing interests}

The authors have no conflicts of interest to declare.

\section{Funding}

The study was supported by the own resources of the University of Rzeszow.

\section{REFERENCES}

1. Finucane MM, Stevens GA, Cowan MJ, Danaei G, Lin JK, Paciorek CJ, et al. National, regional, and global trends in body-mass index since 1980: Systematic analysis of health examination surveys and epidemiological studies with 960 country-years and 9.1 million participants. Lancet. 2011; 377: 557-67. doi: 10.1016/S0140-6736(10)62037-5.

2. Kelly T, Yang W, Chen CS, Reynolds K, He J. Global burden of obesity in 2005 and projections to 2030. Int J Obes. 2008; 32: 1431-7. doi: 10.1038/ijo.2008.102.

3. Global status report on noncommunicable diseases 2010. World Health Organization.http://whqlibdoc.who.int/ publications/2011/9789240686458_eng.pdf (access: 2019.03.20)

4. Ebbert JO, Elrashidi MY, Jensen MD. Managing Overweight and Obesity in Adults to Reduce Cardiovascular Disease Risk. Curr Atheroscler Rep. 2014; 16(10): 445. doi: 10.1007/s11883-014-0445-x.

5. Dhana K, Nano J, Ligthart S, Peeters A, Hofman A, Nusselder W, et al. Obesity and Life Expectancy with and without Diabetes in Adults Aged 55 Years and Older in the Netherlands: A Prospective Cohort Study. PLoS Med. 2016; 13(7): e1002086. doi: 10.1371/journal.pmed.1002086.

6. Castell MV, Van Der Pas S, Otero A, Siviero P, Dennison E, Denkinger $M$, et al. Osteoarthritis and frailty in elderly individuals across six European countries: Results from the European Project on OSteoArthritis (EPOSA). BMC Musculoskelet. Disord. 2015; 16: 359. doi: 10.1186/s12891-015-0807-8.

7. Apostolovic S, Jankovic-Tomasevic R, Salinger-Martinovic S, Djordjevic-Radojkovic D, Stanojevic D, Pavlovic M, et al. Frequency and significance of unrecognized chronic obstructive pulmonary disease in elderly patients with stable heart failure. Aging Clin Exp Res. 2011; 23: 337-42. doi: 10.3275/7291.

8. Arnold M, Freisling H, Stolzenberg-Solomon R, Kee F, O’Doherty MG, Ordóñez-Mena JM, et al. Overweight duration in older adults and cancer risk: a study of cohorts in Europe and the United States. Eur J Epidemiol. 2016; 31(9): 893-904. doi:10.1007/s10654-016-0169-z.

9. Kim J, Noh J-W, Park J, Kwon YD. Body Mass Index and Depressive Symptoms in Older Adults: A Cross-Lagged Panel Analysis. PLoS One. 2014; 9(12): 1-9. doi:10.1371/journal.pone.0114891.

10. Cova I, Clerici F, Maggiore L, Pomati S, Cucumo V, Ghiretti R, et al. Body Mass Index Predicts Progression of Mild Cognitive Impairment to Dementia. Dement Geriatr Cogn Disord. 2016; 41(3/4): 172-180. doi:10.1159/000444216

11. Smit E, Winters-Stone KM, Loprinzi PD, Tang AM, Crespo CJ. Lower nutritional status and higher food insufficiency in frail older US adults. Br J Nutr. 2013; 110(1): 172-178. doi:http://dx.doi.org/10.1017/ S000711451200459X.

12. Suastika K, Dwipayana P, Saraswati MR, Gotera W, Budhiarta AA, Sutanegara ND, et al. Underweight is an important risk factor for coronary heart disease in the population of Ceningan Island, Bali. Diab Vasc Dis Res. 2012; 9(1): 75-77. doi:10.1177/1479164111422828.

13. Che L, Xu L, Wang M-Y, Huang Y-G. Obesity paradox among elderly patients with coronary artery disease undergoing non-cardiac surgery. J Geriatr Cardiol. 2018; 15(9): 598-604. doi:10.11909/j.issn.16715411.2018.09.004.
14. Bottone FG, Ozminkowski RJ, Migliori RJ, Cheng Y, Ozminkowski RJ, Migliori RJ, et al. The relationship between body mass index and quality of life in community-living older adults living in the United States. J Nutr Health Aging. 2013; 17(6): 495-501. doi:http://dx.doi. org/10.1007/s12603-013-0022-y.

15. Pischon T, Boeing H, Hoffmann K, Bergmann M, Schulze MB, Overvad $\mathrm{K}$, et al. General and Abdominal Adiposity and Risk of Death in Europe. N Engl J Med. 2008; 359: 2105-20. doi: 10.1056/NEJMoa0801891.

16. Ferreira LS, do Amaral TF, Marucci M de FN, Nascimento LFC, Lebrão ML, Duarte YA de O. Undernutrition as a major risk factor for death among older Brazilian adults in the community-dwelling setting: SABE survey. Nutrition (Burbank, Los Angeles County, Calif). 2011; 27(10): 1017-1022. doi:10.1016/j.nut.2010.11.008.

17. World Health Organization. Global Health Observatory. Overweight: issues and trends. Available from: http://www.who.int/gho/ncd/risk_ factors/overweight_text/en/ (access: 2019.03.20).

18. World Health Organization. Obesity: preventing and managing the global epidemic: report on a WHO consultation. Geneva, Switzerland: WHO, 2000. http://www.who.int/iris/handle/10665/42330 (access: 2019.03.20)

19. De Hollander EL, Van Zutphen M, Bogers RP, Bemelmans WJE, De Groot LC. The impact of body mass index in old age on cause-specific mortality. J Nutr Health Aging. 2012; 1(16): 100-6.

20. Winter JE, MacInnis RJ, Wattanapenpaiboon N, Nowson CA. BMI and all-cause mortality in older adults: a meta-analysis. Am J Clin Nutr. 2014; 99(4): 875-890. doi:10.3945/ajcn.113.068122.

21. Grzegorzewska A, Wołejko K, Kowalkowska A, Kowalczyk G, Jaroch A, Proper BMI ranges for the elderly in the context of morbidity, mortality and functional status. Gerontol Pol. 2016; 24: 114-118.

22. Mossakowska M, Więcek A, Błędowski P. Aspekty medyczne, psychologiczne, socjologiczne i ekonomiczne starzenia się ludzi w Polsce, część III. Termedia Wydawnictwa Medyczne, 2012.

23. Üstün TB. Measuring Health and Disability: Manual for WHO Disability Assessment Schedule WHODAS 2.0. World Health Organisation 2010;90 https://books.google.com/books?hl=en\&lr=\&i $\mathrm{d}=\mathrm{h} 9 \mathrm{fhLNiaRTgC \& pgis}=1$ (access: 2019.03.20).

24. International Classification of Functioning, World Health Organisation. 2001. https://www.who.int/classifications/icf/icf_more/en/ (access: 2019.03.20).

25. Global Database on Body Mass Index. World Health Organisation. 2015. http://apps.who.int/bmi/index.jsp (access: 2019.03.20).

26. Sirtori A, Brunani A, Liuzzi A, Pasqualinotto L, Villa V, Leonardi M, et al. Quality of life, disability, and body mass index are related in obese patients. Int J Rehabil Res. 2011; 34: 270-2. doi: 10.1097/ MRR.0b013e328347be15.

27. Al Snih S, Ottenbacher KJ, Markides KS, Kuo YF, Eschbach K, Goodwin JS. The effect of obesity on disability vs mortality in older Americans. Arch Intern Med. 2007; 167: 774-80.

28. Williams ED, Eastwood SV, Tillin T, Hughes AD, Chaturvedi N. The effects of weight and physical activity change over 20 years on later-life objective and self-reported disability. Int J Epidemiol. 2014; 43: 856-65. doi: 10.1093/ije/dyu013.

29. Mwanyangala M a, Mayombana C, Urassa H, Charles J, Mahutanga C, Abdullah S, et al. Health status and quality of life among older adults in rural Tanzania. Glob. Health Action. 2010; 3: 36-44. doi: 10.3402/ gha.v3i0.2142

30. Corona LP, Pereira de Brito TR, Nunes DP, da Silva Alexandre T, Ferreira Santos JL, de Oliveira Duarte YA, et al. Nutritional status and risk for disability in instrumental activities of daily living in older Brazilians. Public Health Nutr. 2014; 17(2): 390-395. doi: 10.1017/ S1368980012005319.

31. An R, Shi Y. Body weight status and onset of functional limitations in U.S. middle-aged and older adults. Disabil Health J. 2015; 8(3): 336-344. doi: 10.1016/j.dhjo.2015.02.003.

32. De Stefano F, Zambon S, Giacometti L, Sergi G, Corti MC, Manzato E, et al. Obesity, muscular strength, muscle composition and physical performance in an elderly population. J Nutr Heal Aging. 2015; 19: 785-91. doi: 10.1007/s12603-015-0482-3.

33. Germain CM, Batsis JA, Vasquez E, McQuoid DR. Muscle strength, physical activity, and functional limitations in older adults with central obesity. J Aging Res. 2016; 2016: 8387324. doi: 10.1155/2016/8387324.

34. Peytremann-Bridevaux I, Santos-Eggimann B. Health correlates of overweight and obesity in adults aged 50 years and over: results from the Survey of Health, Ageing and Retirement in Europe (SHARE). SWISS Med Wkly. 2008; 138: 261-6. doi: 2008/17/smw-12067.

35. Gariepy G, Wang J, Lesage A, Schmitz N. Obesity and the risk of disability in a 12-year cohort study: the role of psychological 
distress. Soc Psychiatry Psychiatr Epidemiol. 2011; 46: 1173-9. doi: $10.1100 / 2012 / 167520$

36. Buttery AK, Du Y, Busch MA, Fuchs J, Gaertner B, Knopf H, et al. Changes in physical functioning among men and women aged 50-79 years in Germany: An analysis of National Health Interview and Examination Surveys, 1997-1999 and 2008-2011. BMC Geriatr. 2016; 16(1): 205.

37. Backholer K, Wong E, Freak-Poli R, Walls HL, Peeters A. Increasing body weight and risk of limitations in activities of daily living: A systematic review and meta-analysis. Obes. Rev. 2012; 13(5): 456-468. doi: 10.1111/j.1467-789X.2011.00970.x.

38. Wong E, Woodward M, Stevenson C, Backholer K, Sarink D, Peeter A. Prevalence of disability in Australian elderly: Impact of trends in obesity and diabetes. Prev Med. (Baltim). 2016; 82: 105-10.

39. Maeda K, Shamoto H, Wakabayashi H, Akagi J. Sarcopenia Is Highly Prevalent in Older Medical Patients With Mobility Limitation. Nutr Clin Pract. 2017; 32(1): 110-115. doi: 10.1177/0884533616680355.

40. Zunzunegui MV, Sanchez MT, Garcia A, Ribera Casado JM, Otero A. Body Mass Index and Long-Term Mortality in an Elderly Mediterranean Population. J Aging Health. 2012; 24(1): 29-47. doi: $10.1177 / 0898264311408419$.

41. Rejeski WJ, Marsh AP, Chmelo E, Rejeski JJ. Obesity, intentional weight loss and physical disability in older adults. Obes Rev. 2010;11(9): 671-85. doi: 10.1111/j.1467-789X.2009.00679.x.

42. Lv Y-B, Yuan J-Q, Mao C, Gao X, Yin ZX, Kraus VB, et al. Association of Body Mass Index With Disability in Activities of Daily Living Among Chinese Adults 80 Years of Age or Older. JAMA Network Open. 2018; 1(5): e181915. doi: 10.1001/jamanetworkopen.2018.1915.
43. Cohen-Mansfield J, Shmotkin D, Hazan H. Homebound older persons: Prevalence, characteristics, and longitudinal predictors. Arch Gerontol Geriatr. 2012; 54(1): 55-60. doi: 10.1016/j.archger.2011.02.016.

44. Corica F, Bianchi G, Corsonello A, Mazzella N, Lattanzio F, Marchesini G. Obesity in the Context of Aging: Quality of Life Considerations. Pharmacoeconomics. 2015; 33(7): 655-672. doi: 10.1007/s40273-0140237-8.

45. Oliveira AJ, Rostila M, de Leon AP, Lopes CS. The influence of social relationships on obesity: sex differences in a longitudinal study. Obesity (Silver Spring, Md). 2013; 21(8): 1540-1547. doi: 10.1002/oby.20286.

46. Anis AH, Zhang W, Bansback N, Guh DP, Amarsi Z, Birmingham CL. Obesity and overweight in Canada: An updated cost-of-illness study. Obes Rev. 2010; 11:31-40. doi: 10.1111/j.1467-789X.2009.00579.x.

47. Kearns K, Dee A, Fitzgerald AP, Doherty E, Perry IJ. Chronic disease burden associated with overweight and obesity in Ireland: The effects of a small BMI reduction at population level. BMC Public Health. 2014; 14: 143. doi: 10.1186/1471-2458-14-143.

48. Danaei G. Metabolic mediators of the effects of body-mass index, overweight, and obesity on coronary heart disease and stroke: A pooled analysis of 97 prospective cohorts with 1.8 million participants. Lancet. 2014; 383: 970-83. doi: 10.1016/S0140-6736(13)61836-X.

49. Dhana K, Berghout MA, Peeters A, Ikram MA, Tiemeier H, Hofman A, et al. Obesity in older adults and life expectancy with and without cardiovascular disease. Int J Obes. 2016; 40: 1535-40. doi: 10.1038/ ijo.2016.94.

50. Flicker L, McCaul KA, Hankey GJ, Jamrozik K, Brown WJ, Byles JE, et al. Body mass index and survival in men and women aged 70 to 75 . J Am Geriatr Soc. 2010; 58: 234-41.doi: 10.1111/j.1532-5415.2009.02677.x. 